\title{
COMMISSIONING RUN OF THE LONG-BASELINE NEUTRINO OSCILLATION EXPERIMENT AT THE 12-GEV KEK-PS
}

\author{
Hikaru Sato and the Task Force Team \\ High Energy Accelerator Research Organization (KEK), Tsukuba, 305-0801, Japan
}

\begin{abstract}
A prompt report for the commissioning run of the longbaseline neutrino oscillation experiments will be presented. Improvement of the KEK-PS main ring has been performed for recent years in order to prepare the long-baseline neutrino oscillation experiment. Intensity upgrade studies and the hard ware improvements result in a factor of $50 \%$ increase in the beam intensity to 5-6 $\times 10^{12}$ ppp up to now. It depends on the reduction of the micro-instability at the crossing of the transition energy and the fine tuning of the closed orbit distortion and so on. The neutrino oscillation experiment requests the fast extraction. It needs to construct the fast kicker magnet system, consists of the $12.5 \mathrm{ohm}$ kicker magnets and Blumlein power supplies, and to re-construct the septum magnets and some other equipment. These works have been under going during a long shut down, which was scheduled for the preparation and construction of the neutrino oscillation experiments facility until January, 1999. The switching of the fast extraction and the slow extraction will be possible without the evacuation of the equipment in order to serve the beam for the multi-users as effective as possible. The prompt results of the commissioning run, which will start at the end of January, 1999, and the performance of the improvements of the KEK-PS main ring will be presented.
\end{abstract}

\section{INTRODUCTION}

The KEK-PS complex comprises two $750 \mathrm{keV}$ Cockcroft-Walton pre-injectors, $40 \mathrm{MeV}$ injector linac, $500 \mathrm{MeV}$ booster synchrotron and $12 \mathrm{GeV}$ main ring as shown in Figure 1. It has been operated successfully to serve a proton beam for more than past two decades [1]. Beams have been serving by the half integer slow extraction to East and North counter halls. Beam bunches accelerated in the booster except to the main ring are utilized as NML (Neutron and Meson Laboratory). To meet the need of new physics research, there are several objectives for the PS upgrade. Especially, an intensity upgrade is coming to the urgent problem for the long-baseline neutrino oscillation experiment [2]. This means the fast extracted highintensity beam creates the high current neutrino beam, which will be injected to Super-Kamiokande, about 250 $\mathrm{km}$ west of KEK. The main ring upgrade projects, intensity upgrade study, construction of the fast extraction system have been performed $[3,4,5,6]$. The neutrino beam line and the production target were constructed for same days by the beam channel group in the Institute of Particle and Nuclear Studies [7].

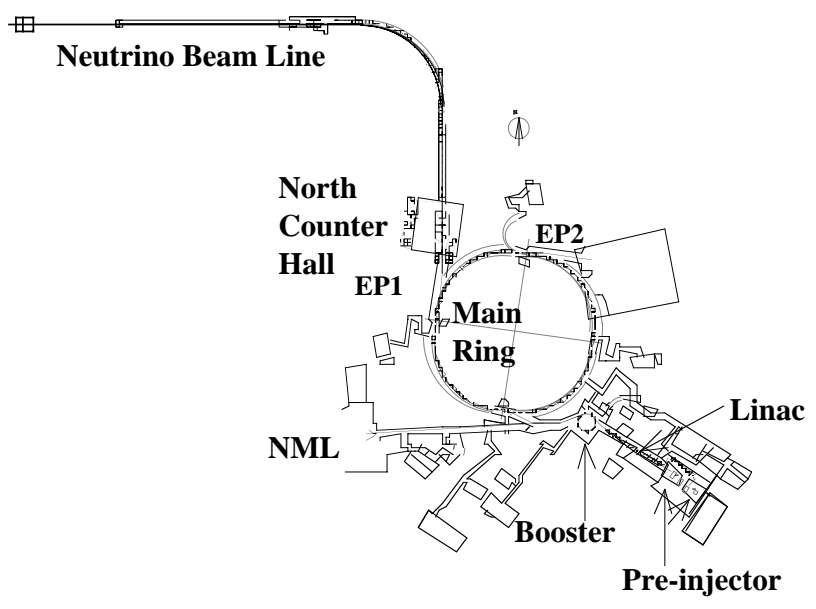

Figure 1 : Layout of the 12-GeV KEK-PS.

\section{INTENSITY UPGRADE STUDIES}

Every effort to realize the upgrade of KEK-PS have been devoted. Booster synchrotron accelerates more than $2 \times 10^{12} \mathrm{ppp}$ for NML, however, the main ring cannot accept the beam of this intensity. Machine studies for the intensity upgrade have continued to make clear the cause and cure of the difficulty. First of all, several tools for the machine study were developed and/or upgraded, such as an injection error monitor, a fast beam loss-monitor, a tune measurement system with an RF kicker, a fast wire scanner and NMR field monitor for the main ring bending magnet.

The concentrated studies in the spring of 1995 was focused on the beam injection to the main ring [3]. It seemed that the forth-order resonance was one candidate and in order to reduce the quadruple imperfection, several correction quadruples were necessary. However there were no installation space in the ring to install them, then the vertical tune was changed from 7.22 to 5.22 to cure the effect of the fourth-order resonance. In order to increase the vertical aperture, the re-alignment of the main ring magnet in the vertical plane was done during summer shut down, 1996. The injection efficiency has increased up to 95- 
97\%. During the magnet realignment was done, the fast floor movement, which seemed to depend on the weather condition, was observed . In order to confirm this phenomena, the measurement of the relative quadrupole magnet level and the floor tilt in the ring were going on [8]. Further, Porous Ceramics Osmometers were set in the soil bank to measure the moisture in the soil under collaboration with Radiation Science Center of KEK and the Geological Survey of Japan.

The loss observed around $80 \mathrm{~ms}$ after the beginning of acceleration results from the horizontal head-tail instability. The instability occurs due to a large change in the chromaticity produced by the sextupole field induced in the beam-pipe of the dipole magnets [4]. In order to suppress this phenomena octu-pole magnets were installed in the main ring and the studies to confirm the suppression of instability and to measure the dynamic aperture are now processing.

Since KEK-PS's impedance budget has been not concerned, a lot of high impedance materials were periodically located along the ring. The one-third of resonant impedance devices, vacuum ducts between the bending magnet and the quadrupole magnet and the beam position monitor, were replaced by low impedance ones in 1996 [5] to care the beam loss at the crossing of the transition. To cross the transition energy in stable condition, $\gamma$-jump magnet power supplies were upgraded to make the voltage twice.

The issues of the intensity upgrade are as follows,

- realignment of the quadrupole magnets in the main ring,

- replacement of bend-quad beam pipe,

- upgrade of beam position monitors,

- installation of beam instrumentation,

- upgrade of the power supply for the $\gamma$-jump magnets,

. installation of octu-pole magnets.

\section{FAST EXTRACTION SYSTEM}

ast extraction of full circulating beam is requested for the neutrino oscillation experiment. The EP1 extraction system was modified so that both of slow and fast extraction are possible. According to the careful orbit analysis for the feasibility using existing slow extraction devices, such as bump and septum magnets system, the changeable system of the extraction kicker and electro-static septum in the same vacuum chamber was decided and the former design, distributed small kickers around in the ring [6], was rejected.

In order to double of the kicker magnetic field and save the transmission time, the Blumlein system was decided to construct [9]. The field strength is requested higher than 0.11T and this should be realized within the space of $3 \mathrm{~m}$ in one long straight section.

The issues of the modification are as follows,

- ESS-kicker chamber construction,

- kicker magnets construction,

- ESS replacement,

- septum A, B magnets improvements,

- septum C magnet power supply construction,

- septum E magnet replacement,

- beam ducts replacement,

- control system upgrade.

\section{NEUTRINO PRODUCTION SYSTEM}

A neutrino beam line extended from EP1-A primary beam line of the north counter hall towards the direction of the Super-Kamiokande as shown in Figure 3. This comprises the straight section, the arc section, the target station, the decay volume and the $\mu$-monitor pit [7]. A fast-extracted proton beam will be transported about $400 \mathrm{~m}$ and focused onto a production target.

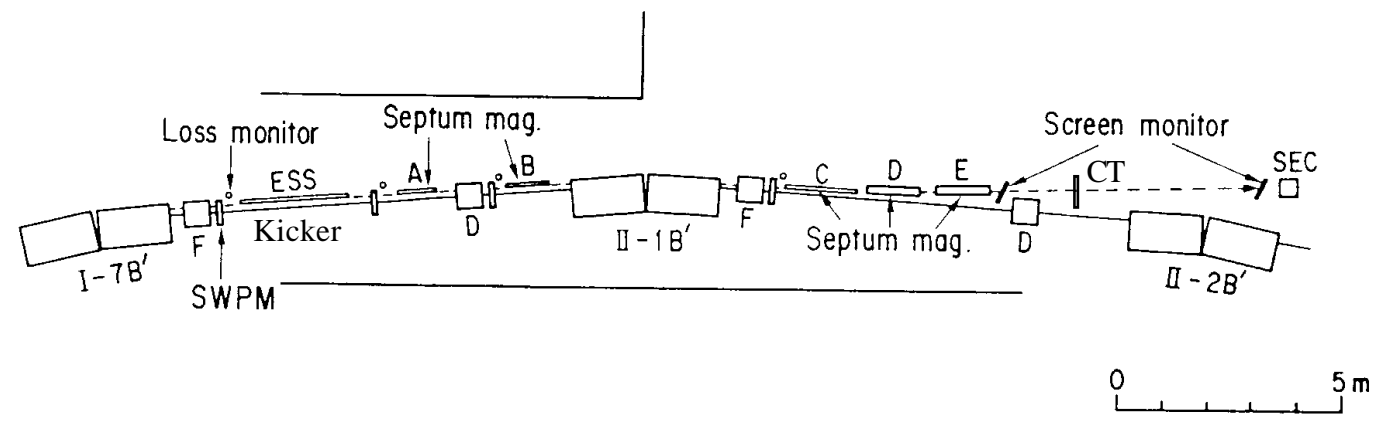

Figure 2: Layout of the EP1 extraction system. ESS and kicker magnets are installed in the same vacuum chamber. Fast extracted beam bunches are measured by $\mathrm{CT}$ at the exit of septum magnet $\mathrm{E}$. 
Two magnetic horns in the target station will focus produced pions to the forward direction, and enhanced by a factor of 14 . The neutrino flux will be produced by decay-in-flight of pions in the $200 \mathrm{~m}$ decay volume. A primary proton beam will be stopped in the beam dump. A high-energy part of muons will be monitored at the $\mu$-monitor pit. A positioning of the Super-Kamiokande from the KEK site was made by the global positioning system (GPS). It is found that the EP 1-A beam line must turn $88^{\circ} 44^{\prime} 25^{\prime}$ ' to the left (west), and bent $10^{\circ} 40^{\prime}$ " downward with respect to the horizontal plane just before the production target [10].

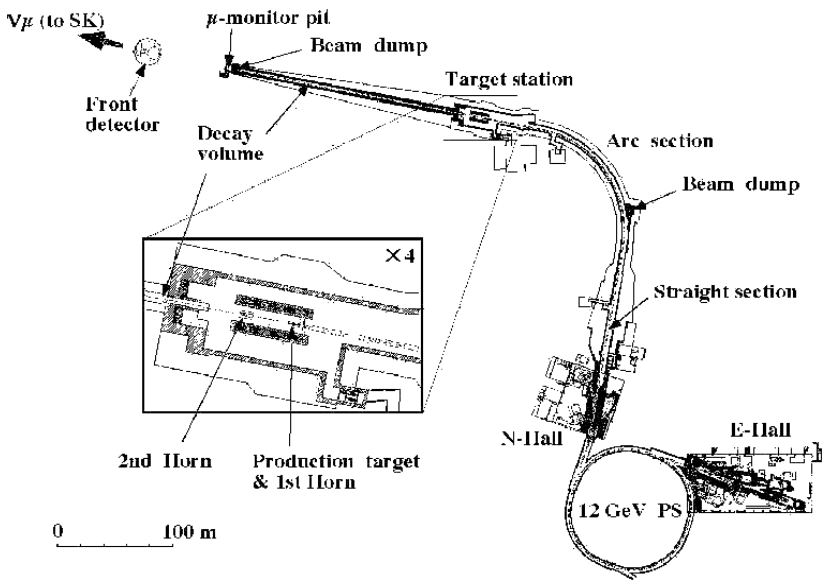

Figure 3 : Layout of the nuetrino beam line and the production target system.

\section{COMMISSIONING RUN}

A fast extracted beam was confirmed on the production target at 16:00 on February 3. Commissioning run was performed as follows.

Main ring beam tuning started on January 27 by the slow extraction mode and the neutrino beam line tuning started on January 31 using slow-extracted beam. On the morning of February 3, the extraction system was changed from the slow extraction mode to the fast extraction mode after tuning the neutrino beam line except the horn system. System change was done in only three hours. Main ring beam tuning started again at 13:04. The kicker magnets were excited and the fast extracted beam was observed on the monitor screen at the exit of the septum magnet E. After short beam-off for the check of some instrumentation, the fast extraction tuning started again and the beam was confirmed on the production target at 16:00. Figure 4 shows the circulating beam bunches, kicker magnet wave form and the extracted beam bunches observed by the current transformer situated at the exit of the septum magnet E. Fast extraction started again on March 3 after the completion of the horn system. The first neutrino event will be expected.

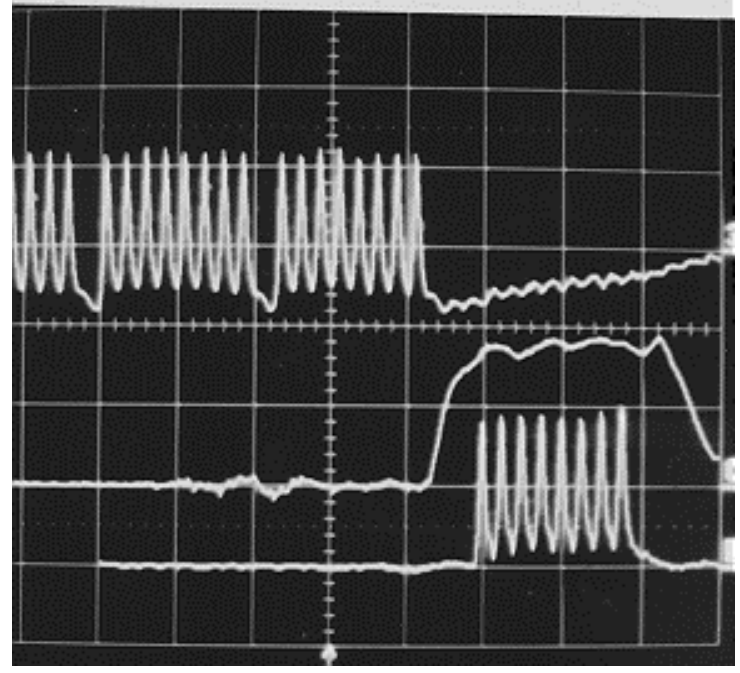

Figure 4 : Beam bunches and the kicker wave form. Upper sweep : Beam current circulating in the ring. One bunch was taken out for the kicker rise.

Middle sweep : Wave form of the kicker magnet. Lower sweep : Extracted beam bunches observed by the CT at the exit of septum magnet E.

\section{ACKNOWLEDGMENTS}

The authors would like to express their sincere thanks to M. Kihara, director general of accelerator laboratory and I. Yamane, director of PS division, for their encouragement. They are much indebted to many colleagues of PS and beam channel staff for their discussions and collaborations.

\section{REFERENCES}

[1] K. Nakai and T. Ohshima, Summary of Experimental Programs at the KEK-PS in KEK-PS 1980's, 1990 and KEK-PS 1990-1994, 1994.

[2] K. Nishikawa et al., KEK Preprint 93-55/INS Report 297-93-9 M. Sakuda, KEK Preprint 97-254, Feb., 1998 (Submitted to APCTP-WS)

[3] Report on the 1995 Spring Machine Study at KEK $12 \mathrm{GeV}$ Proton Synchrotron, KEK Internal 95-12 (in Japanese)

[4] T. Toyama et al., Proc. of the 1997 Part. Accel. Conf., p.1599

[5] K. Takayama et al., Proc. of the 1997 Part. Accel. Conf., p. 1548

[6] H. Sato et al., Proc. of the 1997 Part. Accel. Conf., p.1009

[7] M. Ieiri et al., Proc. of the 1st Asian Part. Accel. Conf., Tsukuba, Japan, 1998, p.579

[8] H. Sato et al., Proc. of the 1st Asian Part. Accel. Conf., Tsukuba, Japan, 1998, p.263

[9] T. Kawakubo et al., Proceedings of the 5th European Particle Accelerator Conference, p.2441

[10] H. Noumi et al., Nucl. Instrum. \& Methods A 398 (1997) 39 